TITLE:

\title{
Time-Resolved Diffusion Detection with Microstopped Flow System
}

$\operatorname{AUTHOR}(S)$ :

Nakasone, Yusuke; Takaramoto, Shunki; Terazima, Masahide

\section{CITATION:}

Nakasone, Yusuke ...[et al]. Time-Resolved Diffusion Detection with Microstopped Flow System. Analytical chemistry 2019, 91(18): 1198711993

ISSUE DATE:

2019-09-17

URL:

http://hdl.handle.net/2433/244208

\section{RIGHT:}

This document is the Accepted Manuscript version of a Published Work that appeared in final form in Analytical chemistry, copyright (c) American Chemical Society after peer review and technical editing by the publisher. To access the final edited and published work see https://doi.org/10.1021/acs.analchem.9b02897.; The full-text file will be made open to the public on 23 August 2020 in accordance with publisher's 'Terms and Conditions for Self-Archiving'.; $こ の$ 論 文は出版社版でありません。引用の際には出版社版をご確認ご利用ください。;This is not the published version. Please cite only the published version. 


\title{
Time-resolved diffusion detection with micro-stopped flow system
}

\author{
Yusuke Nakasone, Shunki Takaramoto, Masahide Terazima* \\ Department of Chemistry, Graduate School of Science, Kyoto University, Kyoto 606-8502, Japan.
}

\begin{abstract}
The transient grating (TG) method is a powerful technique for monitoring the time dependence of the diffusion coefficient during photochemical reactions. However, the applications of this technique have been limited to photochemical reactions. Here, a micro-stopped flow ( $\mu$-SF) system is developed to expand the technique's applicability. The constructed $\mu$ SF system can be used for a solution with a total volume as small as $3 \mu \mathrm{L}$, and mixing times for absorption and diffusion measurements were determined to be $400 \mu \mathrm{s}$ and $100 \mathrm{~ms}$, respectively. To demonstrate this system with the TG method, an acid-induced denaturation of a photosensor protein, phototropin LOV2 domain with a linker, was studied from the viewpoint of the reactivity. This system can be used not only for time-resolved diffusion measurement but also for conventional absorption or fluorescence detection methods. In particular, this system has a great advantage for a target solution in that only a very small amount is needed.
\end{abstract}

The translational diffusion coefficient $(D)$ is an important physical property to study, not only to determine the size of a diffusing molecule but also the conformations (higher-order structures) of proteins and their interaction with the solvent. ${ }^{1}$ There are a tremendous number of studies on $D$ under static conditions. By using these accumulated data, time-resolved measurement of $D$ should be very useful and powerful for revealing the dynamics of chemical reactions, in particular, of protein reactions from the viewpoint of conformational changes and association/dissociation reactions. Although a number of methods, such as Tayler dispersion, tracking a single molecule in a fluorescence microscopy, dynamic light scattering, fluorescence correlation spectroscopy, and nuclear magnetic resonance have been developed, ${ }^{2-6}$ it takes a long time to measure $D$ by these methods, and it is almost impossible to trace the time dependence of $D$. On this point, the pulsed laser induced transient grating (TG) method has unique merits: a high sensitivity and a high time resolution. ${ }^{7-9}$ In the TG method, a target protein is photoexcited by an interference pattern of light (grating light) to create a spatial modulation of molecular concentration, which leads to refractive index modulation in the solution. Then, time development of this modulation is detected by the diffraction of another probe light. Because the spatial modulation of the concentration disappears by the translational diffusion, $D$ can be determined from the rate of the time profile of the signal as shown below. To date, the target reaction has been initiated only by the grating light, which restricts the method to photochemical reactions. Indeed, this TG method has been utilized to study spectrally silent photochemical reactions of various light sensor proteins. ${ }^{7-9}$ In this study, we developed a novel method to overcome this limitation by combining the TG method with a stopped-flow (SF) method. This system expands the applicability of the time-resolved diffusion detection considerably.
An SF instrument is a rapid mixing device, which has been used to study reaction kinetics in solution in a variety of fields. ${ }^{10-12}$ Two (or more) solutions are introduced into a mixing chamber and the flow is stopped suddenly. After the mixing, the reaction dynamics are often monitored by absorption or fluorescence detection techniques. Our strategy here to measure $D$ by the TG method with the SF instrument. After starting a reaction by solution mixing with an SF instrument, the pulsed grating light is irradiated in the observation chamber at a certain delay time, and the TG signal is detected. If the diffusion under the TG conditions is sufficiently fast compared with the mixing period or the reaction rate, we can monitor $D$ at the end of that delay time during the reaction (Fig. 1). By changing the delay time between the mixing and diffusion signal, we can trace the time development of $D$ for reactions started by the solution mixing. In this way, the target reactions that can be studied by the TG technique will be greatly expanded.

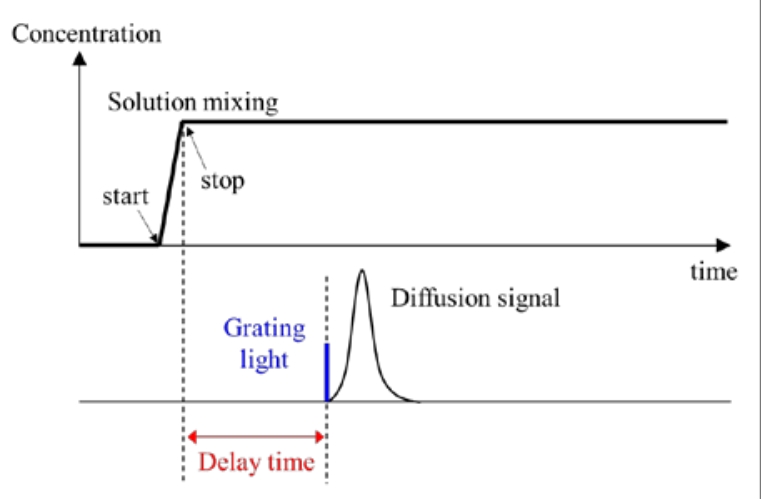

Figure 1. Principle of the SF-TG method. Two solutions are rapidly mixed and stopped suddenly. The stop timing is defined as the starting time of the reaction. After a certain delay time, pulsed grating light is introduced and the 
diffusion signal is detected. Analysis of the time profile reveals the diffusion coefficient at that delay time. Repeating the measurement at various delay times achieves time-resolved diffusion detection.

There are some commercially available SF systems. However, there are mainly two difficulties to use these systems for the SF-TG measurement. First, because the TG signal should be created by pulsed grating light and monitored in the time domain, we cannot monitor the reaction continuously in the mixing chamber, and hence, the TG signals should be detected at various delay times after mixing to monitor the time dependence of $D$. Furthermore, if we need to average the signal to improve the signal-to-noise ratio, the TG measurements should be repeated for each delay time to accumulate the signals. Consequently, the sample solution should be mixed many times, and generally the mixed solution cannot be used again. Moreover, the amount of protein solution that is available is generally very small, particularly for biological proteins. Hence, it is essential to make the mixing volume very small. Second, the convection flow created by the rapid mixing disturbs the $D$ measurement. The solution inside the observation cell should stop as quickly as possible to obtain a fast time resolution. To overcome these difficulties, we newly developed a micro-SF ( $\mu$-SF) system.

To demonstrate the application of the SF-TG method, we investigated acid-induced denaturation of a light sensing protein from the viewpoint of loss of reactivity. Phototropin (phot) is a blue light sensor protein in plants. There are two phots (phot1 and phot2) in Arabidopsis thaliana and these phots consist of two light-oxygen-voltage domains (LOV1 and LOV2) and a serine/threonine kinase domain. ${ }^{13}$ The LOV domains are light-sensing modules. The LOV domain binds a flavin mononucleotide (FMN) as a chromophore, and its excitation leads to an adduct formation with a nearby cysteine residue on a microsecond timescale. ${ }^{14}$ This reaction of the LOV2 domain triggers subsequent conformation changes of the linker domain to decrease $D$. Detailed analyses of diffusion signals revealed that the $D$ change of phot1 LOV2 with the linker domain from Arabidopsis thaliana (LOV2-linker) occurs with a time constant of 1 ms upon photoexcitation, which is attributed to conformation change of the helical structure in the linker domain (Fig. S1). ${ }^{15}$ This reaction cannot be detected by an absorption measurement. We tried to detect $\mathrm{pH}$-induced unfolding process of LOV2linker by monitoring $D$. Because $D$ reflects the conformation and the signal intensity represents the reactivity of the protein, it is expected that the protein unfolding process can be detected from the viewpoint of the conformation and reactivity in the time domain. We found that the reactivity decreased in two steps with time constants of less than $100 \mathrm{~ms}$ and $13.2 \mathrm{~s}$, which were assigned to a local structural change and further denaturation that accompanies a dissociation of the chromophore, respectively.

\section{Experimental Method}

$\mu$-SF apparatus. A T-junction type micromixer made of quartz is shown in Fig. 2a. The diameter of the mixing part is $0.46 \mathrm{~mm}$ (Namiki Seimitsu Co.). An observation cell was a rectangular hole with dimensions of $0.8 \times 0.8 \times 3.0 \mathrm{~mm}$ (Japan Cell). Flow tubes are connected to the hole of the mixer (diameter $=1.6 \mathrm{~mm}$ ) using a handmade jig (Fig. $2 \mathrm{~b}$ ). This component was placed on a stage to finely adjust the position for measurements.

Fluid flow in a narrow path tends to be laminar, which inhibits efficient mixing of two solutions. To avoid laminar flow, high-speed flow is required for generating turbulence. To achieve the high-speed flow, a pump-valve system (Fig. 2c) was developed. The sample solution was introduced into the observation cell with high-pressure compressed air. The pressure was increased to 2.5 bar using a compressor (Nitto Kohki, DP0105-Y1-0001) and further compressed by a plunger pump (Lee Co., LPVA1700330H). This system withstands high operating pressure up to 6.0 bar. The pressure was monitored by a pressure sensor (Schneider Electric, XMLP010BD71V).

The timing of the starting and stopping of the flow was regulated with high time resolution and high precision by four two-way valves (valves 1-4: Takasago Electronics, MTV-2SL-N32UF) (Fig. 2c). These valves open and close very fast even at high pressure. The internal volume associated with the open/close switching did not change, and movement of solution due to the switching was negligible. To fill the flow paths with solution, two three-way valves were set as shown in Fig.2c. All valves shown in Fig. $2 \mathrm{c}$ were controlled by a computer.

Because the valves used here could not respond to sequential triggers that control the opening and closing timing within a very short period, two valves (valve 1 and 2) were used. Each valve has a role of opening (valve 2) or closing (valve 1) sequentially to achieve a short opening period with high precision. The amount of flow was regulated by changing the opening time. Valves 3 and 4 were used to prevent the movement of the solution inside the flow path. After the mixing, a triggering pulse with a certain delay time was sent to a pulsed laser to control the monitoring time for the TG signal. A Radel tube, which has high-pressure resistance (37.9 MPa), with an outer diameter of $1.6 \mathrm{~mm}$ and an inner diameter of $0.76 \mathrm{~mm}$ was used for the pumping tube.

The experimental procedure was the following:

1. Fill flow paths: Valves 1-4 were opened and three-way valves were connected to the sample pools. A syringe pump (YMC Corp., YSP-202) was used to load the flow paths with solutions A and B.

2. Compress air: Three-way valves were switched to connect air and flow path, and only valve 2 was closed. The air was compressed by the compressor and the plunger pump. The pressure was finely controlled using this plunger pump.

3. Start mixing: Valve 2 was opened. The sample solutions were pushed into the micromixer.

4. Stop mixing: Valves 1, 3, and 4 were closed simultaneously to stop flow.

5. Take measurement: The TG signal at a set delay time after stopped flow was detected. For an absorption measurement, the transmitted light intensity was recorded after mixing stopped. 
(a)

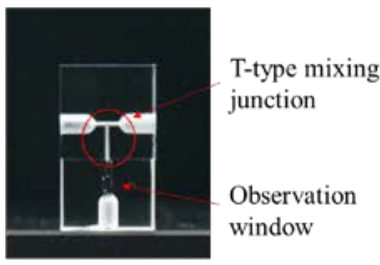

(b)

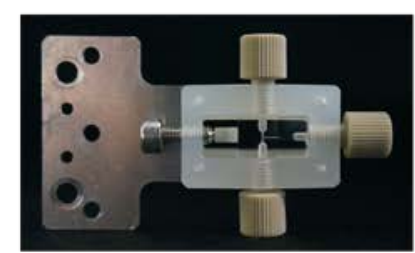

(c)

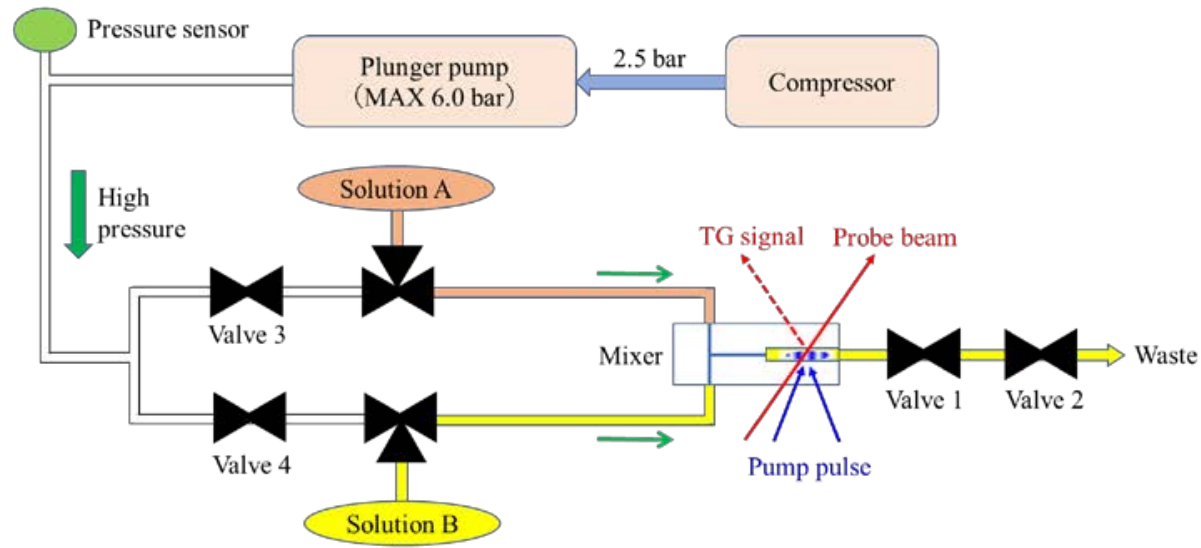

Figure 2. (a) Photograph of the micromixer. (b) Photograph of the jig. (c) Schematic diagram of the $\mu$-SF apparatus for the SF-TG measurement.

6. Restore the pre-measurement state: After the measurements, valve 2 was closed and then valves 1,3 , and 4 were opened.

7. Repeat measurement: Steps 3-6 were repeated until the loaded sample ran out.

Usually, it was possible to perform about 30 shots of mixing by one load of the solution in the pumping tube.

During the period between step 2 and 6, two reactant solutions are in contact and they are mixed gradually by diffusion at the T-junction. However, since the diffusion is a rather slow process, the mixed volume could be very small. For example, if the SF-TG measurements are repeated with a waiting time of $30 \mathrm{~s}$, the mixing at the Tjunction by the diffusion is $c a .80 \mu \mathrm{m}$ for a small protein having a diffusion coefficient of $1.0 \times 10^{-10} \mathrm{~m}^{2} / \mathrm{s}$. Even if a part of two solutions are mixed by convection, the mixing does not seriously disturb the measurement, because the sample cell is completely refreshed by each mixing step due to much larger flow volume. Hence, the effect of the mixing during the period between step 2 and 6 is negligible.

The solution mixing process was recorded by using a high-speed camera (Photron, FASTCAM SA-X2).

Preparation of samples. LOV2-linker of phototropin 1 from Arabidopsis thaliana (residue 449E-661K) was purified as previously reported. ${ }^{16}$ Concentrations of the proteins $(100 \mu \mathrm{M})$ were determined by absorbance at $447 \mathrm{~nm}$ (extinction coefficient of $14,300 \mathrm{M}^{-1} \mathrm{~cm}^{-1}$ ). ${ }^{17}$ In all spectroscopic measurements, we used PBS buffer (10 $\mathrm{mM} \mathrm{Na}_{2} \mathrm{HPO}_{4}, 1.8 \mathrm{mM} \mathrm{KH}_{2} \mathrm{PO}_{4}, 137 \mathrm{mM} \mathrm{NaCl}$, and $2.7 \mathrm{mM}$ $\mathrm{KCl}$ ). Solution $\mathrm{pH}$ was adjusted with hydrochloric acid.

TG measurement. The experimental setup for the TG measurement was similar to that reported previously. ${ }^{15}$ A laser pulse from a dye laser (Lumonic, HyperDye 300; wavelength, $462 \mathrm{~nm}$ ) pumped by an excimer laser (Lambda Physic, XeCl operation; $308 \mathrm{~nm}$ ) was used as an excitation beam for the creation of the TG. A diode laser $(835 \mathrm{~nm})$ was used as a probe beam. All measurements were carried out at $23^{\circ} \mathrm{C}$.

Transient absorption measurement. Absorption change due to the adduct formation between FMN and Cys residue was monitored by the same pulse laser as the
TG method. A diode laser (449 $\mathrm{nm}$ ) was used as a probe beam.

Principle of TG method. The principle of the TG method has been reported before. ${ }^{1}$ The TG signal is a probe light diffracted by a refractive index grating. The signal intensity is proportional to the square of the refractive index change by the excitation light. If a photochemical reaction completes before an observation time range, the time profile of the TG signal after the thermal grating component may be given by

$I_{T G}(t)=\alpha\left\{-\delta n_{R} \exp \left(-D_{R} q^{2} t\right)+\delta n_{P} \exp \left(-D_{P} q^{2} t\right)\right\}^{2}$,

(1)

where $\alpha$ is a constant; $q$ is the grating wavenumber; $D$ is the diffusion coefficient; $\delta n(>0)$ is the initial refractive index changes; and subscripts $R$ and $P$ represent the reactant and product, respectively. If $D$ of the product is the same as that of the reactant, the signal decays single exponentially. If the reaction changes $D$, the bi-exponential behavior of the signal usually gives rise to a characteristic rise-decay profile. The signal component representing the diffusion process is referred to as the diffusion signal.

\section{Results}

Performance of $\mu$-SF system. To check the mixing efficiency in the micromixer, we recorded the flow using a high-speed camera. Here, we monitored the color change of an aqueous solution of bromocresol purple (BCP) induced by $\mathrm{pH}$ changes. The $200 \mu \mathrm{M}$ BCP solution in distilled water at $\mathrm{pH} 6.5$ was mixed with a $100 \mathrm{mM}$ carbonate buffer $(\mathrm{pH} 10)$. The color of BCP solution is changed from yellow to violet immediately by the increased $\mathrm{pH}$. Movie S1 (pressure $=4.0 \mathrm{bar}$, frame rate $=$ $40,000 \mathrm{fps}$, total time $=18 \mathrm{~ms}$, total amount of solution flowed $=6.0 \mu \mathrm{L}$ ) shows that turbulence is generated during the mixing, and two solutions are mixed very efficiently. The flow rate in the mixing area was calculated to be faster than $10 \mathrm{~m} / \mathrm{s}$ from the flow volume and the valve open period. Using this flow rate, the viscosity of the solution and the diameter of flow path, the Reynolds number was calculated to be higher than 4,600 . This value is 
much higher than the threshold for generating turbulence $(R e=2,000)$. Because we found that $4.0 \mathrm{bar}$ is high enough to achieve efficient mixing, the pressure of $4.0 \mathrm{bar}$ was used in subsequent experiments.

The reproducibility of the mixing ratio of the $\mu$-SF system was tested as follows. The FMN solution $(900 \mu \mathrm{M})$ was mixed with the same amount of water (i.e., $50 \%$ dilution) and this dilution experiments were repeated 27 times for one loaded solution in the pumping tube, and this measurement was repeated by 24 sets. From the transmitted light intensity after the mixing, the mixing ratios were determined and shown in Figure S2. An averaged mixing ratio was deviated only $1.3 \%$ from the expected $1: 1$ ratio and its standard deviation was $1.9 \%$. The stability of mixing volume was also examined and described in Figure S3, although stability of this mixing volume is not an important factor for the SF measurement. The mixing ratio slightly depends on the difference in the viscosities of two solutions, probably because the flow rate should be slower for viscous solution. For example, if we used two solutions having different viscosities (1.0 $\mathrm{mPa} \cdot \mathrm{s}$ and $1.3 \mathrm{mPa} \cdot \mathrm{s}$ ), the mixing ratio slightly changed about $3.7 \%$ compared with the two solutions having the same viscosity (Figure S4). A correction of this difference may be needed, when we will use two solutions having different viscosities.

For a time-resolved measurement, the system response time is important. In this SF-TG method, there are two types of system responses. One is the mixing time of two solutions and the other is response time for diffusion measurement. The mixing time was measured by monitoring the time profile of an absorption change due to solution mixing. The absorption change of 2,6-dichlorophenolindophenol (DCIP) by mixing with ascorbic acid (reductive reaction) was monitored. Fig. 3a shows the absorption spectra of DCIP in the absence and presence of ascorbic acid. When DCIP is reduced by ascorbic acid, the absorption band in the visible region disappears thoroughly. Fig. $3 \mathrm{~b}$ shows the absorption change at $532 \mathrm{~nm}$ after mixing of $500 \mu \mathrm{M}$ DCIP solution and various concentrations of ascorbic acid ([ascorbic acid]) of $1 \mathrm{mM}-40$ $\mathrm{mM}$. As the [ascorbic acid] was increased, the rate constant of the absorption change was increased. All signals are reproduced by a single exponential function and the determined rate constant $\left(k_{\mathrm{obs}}\right)$ was plotted against the final concentration of ascorbic acid in Fig 3c. The linear correlation between $k_{\text {obs }}$ and the [ascorbic acid] indicates that the reaction is the pseudo-first order and we determined the second-order rate constant from the slope of the plot to be $5.2 \pm 0.5 \times 10^{4} \mathrm{M}^{-1} \mathrm{~s}^{-1}$. Because this value is almost the same as the reported value $\left(5.6 \times 10^{4} \mathrm{M}^{-1} \mathrm{~s}^{-}\right.$ $\left.{ }^{1}\right),{ }^{18,19}$ we consider that this $\mu$-SF system can mix two solutions with a precise ratio. Importantly, the sample consumption for mixing was only $3 \mu \mathrm{L}$ and the dead time for the absorption measurement was estimated to be $400 \mu \mathrm{s}$. The sample consumption volume is very small compared with that of a commercial SF system. Once the tubes (30 $\mathrm{cm}$ long) are filled with the sample solution, about $30 \mathrm{se}-$ quential measurements are possible. During the repeated measurements, the pressure of the air was kept at 4.0 bar by fine adjustment of the plunger.
In many cases of SF experiments, complete mixing of two solutions with different viscosities is difficult. For our $\mu$-SF system, we also found that the mixed solution was not uniform just after the stopping of the flow of two solutions with different viscosities (Figure S4). To overcome this problem, a modification of the mixer to increase the turbulence is necessary, and such system will be reported in future.
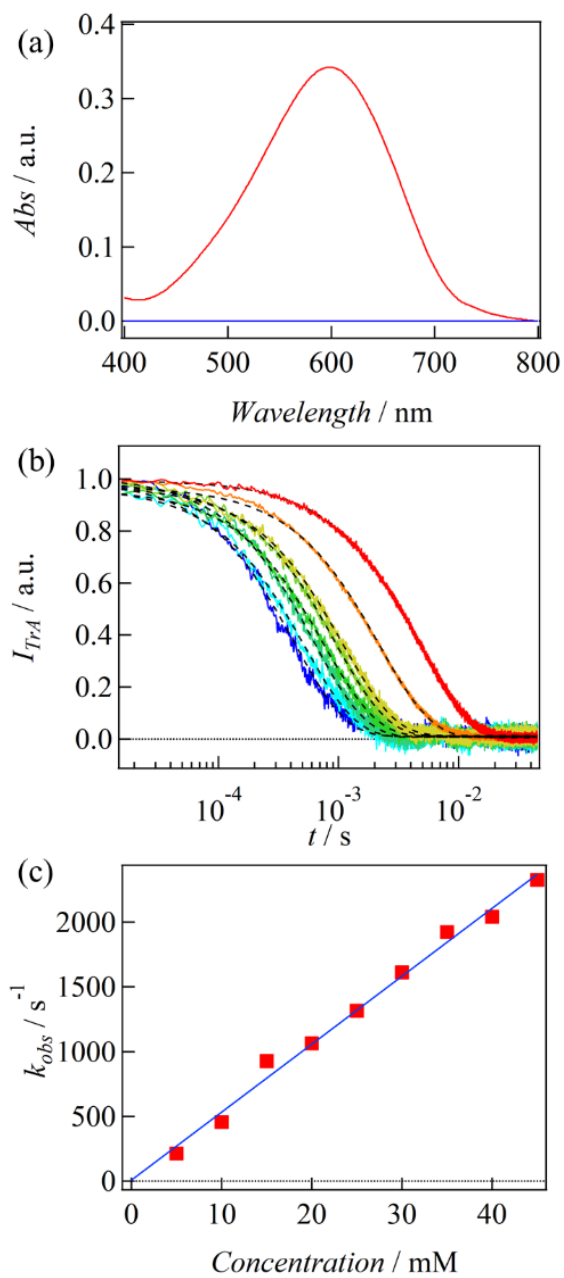

Figure 3. (a) Absorption spectra of DCIP in oxidized (red) and reduced (blue) forms. (b) The absorption change monitored at $532 \mathrm{~nm}$ after mixing with various concentrations of ascorbic acid in the $\mu$-SF system. Fitted curves from a single exponential function are shown (black broken lines). (c) The apparent rate constant of the absorption change is plotted against the final concentration of ascorbic acid. The plot is fitted by a linear function (blue line).

The response time for the diffusion measurement was examined by using the diffusion signal. For this purpose, a reaction of LOV2-linker was used. The reaction scheme, as well as the TG signal in a static neutral $\mathrm{pH}$ solution, have been reported previously, ${ }^{15}$ and it was found that $D$ decreases with a time constant of $1 \mathrm{~ms}$ after the photoexcitation. The molecular origin of the decrease in $D$ is attributed to the conformation change of the linker region. In the following discussion, we used a relatively low $q$ to 
observe the diffusion signal, and the diffusion signal was fitted by Eq. 1.

Figure 4 shows the diffusion signal of LOV2-linker obtained at $q^{2}=1.2 \times 10^{12} \mathrm{~m}^{-2}$ under various delay times after the mixing of two identical solutions. When the delay time was shorter than $100 \mathrm{~ms}$, the diffusion signal shifted to a faster time scale and its intensity considerably decreased due to the solution convection. However, when the delay time was longer than $100 \mathrm{~ms}$, the diffusion signal of LOV2-linker was almost identical to that obtained without solution mixing. Therefore, the response time of the diffusion measurement was determined to be about $100 \mathrm{~ms}$ for this protein system.

The slower response time compared to that of absorption measurement is mainly due to the effect of the convection flow. For the diffusion measurement, the mixed solution should not move including the convection flow. On the other hand, the absorption measurement is not disturbed by the movement (or even flow) of the solutions as long as the solution is homogeneous. Therefore, the response time for the diffusion measurement is slower than that for absorption measurement.

We consider that, in addition to an accurate and instantaneous regulation of flow using electronic valves, the downsizing of the mixer is important for achieving rapid stopping of the convection flow in the observation cell, because friction between the cell wall and solution should be important for stopping the convection flow. Indeed, comparing with a commercially available SF system (Applied photophysics, RX2000), which has a relatively larger observation cell with a dimension of $2.0 \times 2.0 \times 10.0$ $\mathrm{mm}$, we found that the response time for the diffusion measurement is improved more than a factor of 10 by using this $\mu$-SF system (Figure 4 and Figure S5).

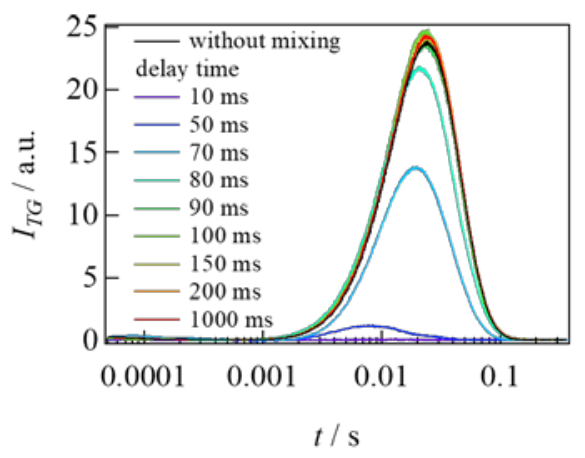

Figure 4. The diffusion signals of LOV2-linker obtained at several delay times after mixing two identical solutions containing $100 \mu \mathrm{M}$ protein using the $\mu$-SF system $\left(q^{2}\right.$ $=1.2 \times 10^{12} \mathrm{~m}^{-2}$ ). The weaker signal at the short delay time indicates that the concentration modulation of the protein is perturbed by convection caused by the solution mixing. The dead time for the diffusion measurement is estimated to be $100 \mathrm{~ms}$.

Acid-denaturation kinetics of LOV2-linker. By using the $\mu$-SF and TG system described above, a kinetic trace of acid denaturation of LOV2-linker was measured using the diffusion signal. In this SF-TG experiment, LOV2linker solution $(100 \mu \mathrm{M}$ in a PBS buffer, $\mathrm{pH} 7.5)$ was mixed with a PBS buffer at $\mathrm{pH}$ 1.5. Upon mixing of the equivalent amount of the two solutions, the $\mathrm{pH}$ decreased rapidly from 7.5 to 2.5 . We performed the TG measurements $\left(q^{2}=1.1 \times 10^{12} \mathrm{~m}^{-2}\right)$ at various delay times after the $\mathrm{pH}$ jump and found that the intensity of the diffusion peak decreased with increasing delay time, as shown in Fig. 5a. For comparison, the TG signal of the native form (absence of denaturation) was measured under the same setup after mixing the $100-\mu \mathrm{M}$ LOV2-linker solution ( $\mathrm{pH} 7.5$ ) with the PBS buffer at $\mathrm{pH}$ 7.5.

(a)
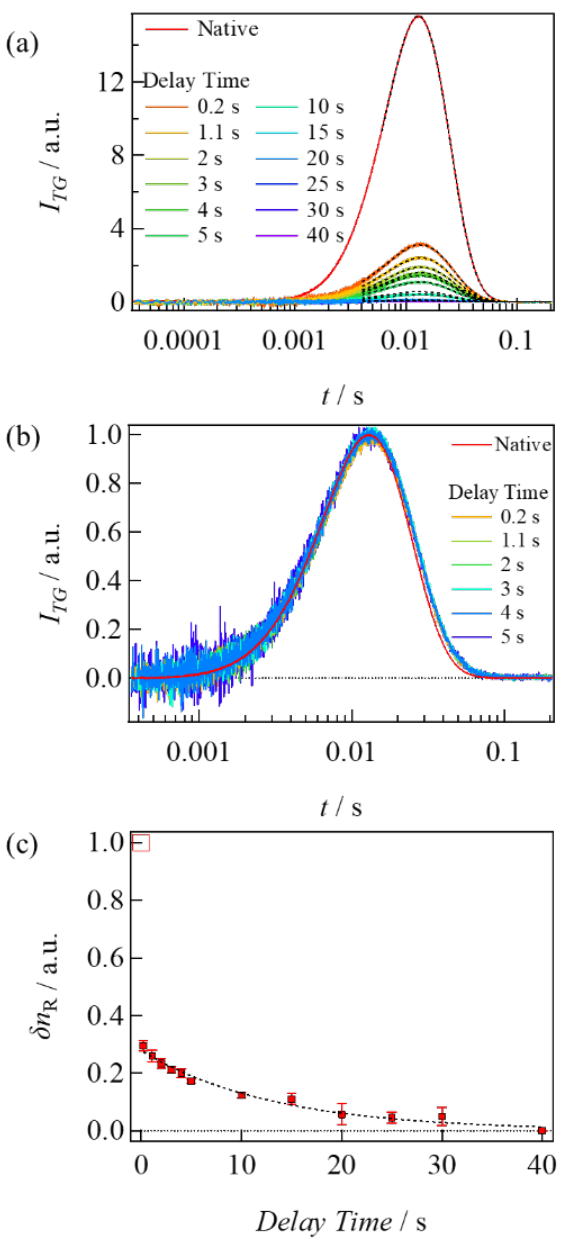

Figure 5. (a) TG signals obtained by the SF-TG measurement system during the acid denaturation of LOV2-linker $\left(q^{2}=1.1 \times 10^{12} \mathrm{~m}^{-2}\right)$. The delay times after the solution mixing are indicated in the legend. The signal of the native form obtained under the same conditions is also shown. The fitting curves based on Eq. 1 are shown as a dotted line. (b) The signals are normalized with the intensity of the diffusion signal. (c) The refractive index of the reactant $\left(\delta n_{\mathrm{R}}\right)$ obtained by the analyses using Eq. 1 is plotted against the delay time (closed squares). That of the native state is shown by an open square. The time dependence of the closed squares is fitted by a single exponential function and shown as a dotted line.

According to Eq. 1, the intensity of the diffusion signal changes depending on the ratio of $D_{R}$ to $D_{P}\left(D_{\mathrm{P}} / D_{\mathrm{R}}\right)$ and/or the number of photoreacted molecules. If the ratio of $D$ changes during the denaturation, the temporal profile of the molecular diffusion signal should change. When the 
signals are normalized, however, the temporal profiles are almost identical, as shown in Fig. 5b, except for the signal of the native form. Hence, the decrease in the signal intensity with the delay time is not due to the change in $D_{\mathrm{P}} / D_{\mathrm{R}}$ but due to the decrease in the number of reactive molecules by the denaturation.

$D_{\mathrm{R}}$ and $D_{\mathrm{P}}$ of native form were previously determined to be $9.2 \pm 0.4 \times 10^{-11} \mathrm{~m}^{2} / \mathrm{s}$ and $6.2 \pm 0.3 \times 10^{-11} \mathrm{~m}^{2} / \mathrm{s}$, respectively. ${ }^{20}$ Diffusion signals obtained at various delay times (0.2-30) are globally fitted by Eq. 1 . For avoiding ambiguity in the fitting, $D_{\mathrm{R}}$ was fixed $\left(9.2 \times 10^{-11} \mathrm{~m}^{2} / \mathrm{s}\right)$ and $D_{\mathrm{P}}$ was determined to be $5.2 \pm 0.3 \times 10^{-11} \mathrm{~m}^{2} / \mathrm{s}$. It is interesting to note that $D_{\mathrm{P}}$ is smaller in the acid condition. This result suggests that the structure of the reactant (conformation in the dark state) is maintained even at a low $\mathrm{pH}$, but the degree of conformation change of the $\alpha$-helices in the linker region is enhanced at low pH. Fig. $5 \mathrm{c}$ shows the time course of $\delta n_{\mathrm{R}}$ obtained by the global analysis. The number of reactive molecules decreased significantly within the response time of the SF-TG $(<100 \mathrm{~ms})$, which was followed by a further decrease with a time constant of $13.2 \pm 1.4 \mathrm{~s}$.

To explore the effect of the sudden $\mathrm{pH}$ increase on the photochemistry of the chromophore FMN, we measured the absorption change caused by the adduct formation process. Fig. S6a shows the transient absorption signals obtained for the native state and at a delay time of $100 \mathrm{~ms}$. The decreases of absorption at the wavelength of the probe beam $(449 \mathrm{~nm})$ are almost identical to each other, indicating that the photochemistry of the chromophore is maintained at this delay time. Therefore, in the early stage of the acid denaturation ( $<100 \mathrm{~ms})$, not the photoreaction of the chromophore, but the signaling path between the chromophore and the linker helix is disrupted.

The pH-dependent absorption spectrum of LOV2linker is shown in Fig. S6b. In the native form, an absorption peak $(449 \mathrm{~nm})$ in the blue region with prominent vibrational bands ( $424 \mathrm{~nm}$ and $474 \mathrm{~nm}$ ) is observed. Under low $\mathrm{pH}$ conditions, however, the vibrational bands disappear and the peak position is blue-shifted slightly. This spectrum is very similar to that of the absorption spectrum of FMN in solution (Fig. S6c). This feature strongly indicates that the acid denaturation causes dissociation of FMN from the LOV2 domain interior. To detect the dissociation kinetics of the chromophore, the absorption at $380 \mathrm{~nm}$ was traced after the $\mathrm{pH}$ increase (Fig. S6d). The time constant of the absorption change was determined to be $15.8 \pm 0.6 \mathrm{~s}$, which is similar to the slower time constant detected by the SF-TG method. Therefore, we conclude that the slower change in the diffusion signal intensity results from the dissociation of FMN from the binding pocket of the LOV2 domain by acid denaturation.

CD spectra of LOV2-linker were measured at various $\mathrm{pH}$, and it was found that the amount of the helical structure (CD intensity at $220 \mathrm{~nm}$ ) is partially decreased but not completely within this $\mathrm{pH}$ range (Figure S7a). The pKa of the unfolding of the helical structure was determined to be 3.0 (Figure S7b). We consider that a part of secondary structure of this protein has a rather high resistance to the acid condition. Utilizing the SF-TG method, we can clearly detect the denaturation process as a loss of reactivity even though the acid induced CD change is relatively minor.

Although a detailed analysis of the observed denaturation process is not the main target in this paper, we briefly describe possible molecular origins. The acidic stress locally denatures the LOV2-linker in the early stage of denaturation, which results in a considerable loss of the relaying signal from FMN to the linker helix. An molecular dynamics simulation on phot1LOV2 from Avena sativa has reported that the disruption of a hydrogen bond between Gln497 in H $\beta$ and Asp540 in the linker helix is observed in the light state, which causes partial unfolding of the linker helix. ${ }^{21}$ Because phot1LOV2 from $\mathrm{Ar}$ abidopsis thaliana has the same residues (Gln559 and Asp609) at the same location (Fig. S8), weakening of the hydrogen bond may be induced by reduced $\mathrm{pH}$. This possibly prevents photoinduced unfolding of the helices in the linker region.

The slower change with the time constant of $13.2 \mathrm{~s}$ is assigned to the dissociation of chromophore from the binding pocket of the LOV domain. The disruption of a salt bridge between Glu506 and Lys547 (Fig. S8) on the surface of the LOV2 domain might be relevant. Previous work reported that the formation of the tertiary structure that packs the FMN requires this ionic interaction. ${ }^{22}$ If the protonation of this surface residue is the only origin of the dissociation of the chromophore, it should be a fast reaction because the protonation occurs quite fast. However, the observed rate of dissociation is slow. This slow change indicates that the structure of the LOV2 domain is rather stable even in the acidic condition, and hydrophobic interaction between FMN and binding pocket slows down the rate of dissociation.

\section{Conclusions}

We have newly developed the SF-TG method, which can detect protein dynamics from the viewpoint of diffusion change. The $\mu$-SF system requires $3 \mu \mathrm{L}$ of protein solution for mixing, and dead times for absorption and diffusion measurement were determined to be $400 \mu \mathrm{s}$ and $100 \mathrm{~ms}$, respectively. We investigated the denaturation process of photosensor protein LOV2-linker using the system and found that the reactivity was significantly lost within $100 \mathrm{~ms}$, even though the photochemistry of the chromophore was not affected. This demonstrates that the diffusion probe is highly sensitive to conformational changes in the protein part, which should be relevant to its function. Hence, this system will be a promising tool to study the folding and unfolding reactions of proteins. In addition, this system will be used for various kinds of protein reactions, such as enzymatic reactions, signaling, and complex formations, by the time-resolved diffusion technique. Of course, this system can be used not only for time-resolved diffusion detection but also for conventional absorption or fluorescence detection methods. It is particularly useful for target solutions that we can prepare only in very small amounts.

\section{ASSOCIATED CONTENT}

\section{Supporting Information}


Supporting information is available free of charge on the ACS Publications website.

This information includes the crystal structure and typical TG signal of LOV2-linker, reproducibility of mixing ratio of the $\mu$ SF system, reproducibility of mixing volume of the $\mu$-SF system, effect of different viscosities on mixing rate and mixing ratio, response time of the RX2000 for diffusion measurement, transient absorption signals of LOV2-linker, $\mathrm{pH}$-dependent absorption spectra of LOV2-linker, absorption spectrum of free FMN, kinetic trace of absorption at $380 \mathrm{~nm}$ after $\mathrm{pH}$ jump, pH-dependent CD spectra of LOV2-linker, and mapping of key residues for signal transduction and binding of FMN (PDF).

\section{AUTHOR INFORMATION}

\section{Corresponding Author}

mterazima@kuchem.kyoto-u.ac.jp

\section{ACKNOWLEDGMENT}

The authors are indebted to Mr.T.Yoshitake for his assistance in some experiments. This work was supported by Grants-inaid for Scientific Research on Innovative Areas (research in a proposed research area) (Nos. JP20107003 and JP25102004) from the Ministry of Education, Culture, Sports, Science and Technology and Grants-in-aid for Scientific Research from the Japan Society for the Promotion of Science (25288005 and 17 H03008 to M.T., 17 H05001 and 18 H04552 to Y.N.).

\section{REFERENCES}

(1) Terazima, M. Diffusion Coefficients as a Monitor of Reaction Kinetics of Biological Molecules. Phys. Chem. Chem. Phys. 2006, 8, 545-557.

(2) Alizadeh, A.; Nieto de Castro, C. A.; Wakeham, W. A. The Theory of the Taylor Dispersion Technique for Liquid Diffusivity Measurements. Int. J. Thermophys. 1980, 1, 243-284.

(3) Stetefeld, J.; McKenna, S. A.; Patel, T. R. Dynamic Light Scattering: A Practical Guide and Applications in Biomedical Sciences. Biophys. Rev. 2016, 8, 409-427.

(4) Elson, E. L. Fluorescence Correlation Spectroscopy: Past, Present, Future. Biophys. J. 2011, 101, 2855-2870.

(5) Price, W. S. Pulsed-Field Gradient Nuclear Magnetic Resonance as a Tool for Studying Translational Diffusion: Part 1. Basic Theory. Concepts Magn. Reson. 1997, 9, 299-336.

(6) Kamagata, K.; Murata, A.; Itoh, Y.; Takahashi, S. Characterization of Facilitated Diffusion of Tumor Suppressor P53 along DNA Using Single-Molecule Fluorescence Imaging. $J$. Photochem. Photobiol. C Photochem. Rev. 2017, 30, 36-50.

(7) Terazima, M. Time-Dependent Intermolecular Interaction during Protein Reactions. Phys. Chem. Chem. Phys. 2011, 13, 16928.

(8) Terazima, M. Studies of Photo-Induced Protein Reactions by Spectrally Silent Reaction Dynamics Detection Methods: Applications to the Photoreaction of the LOV2 Domain of Phototropin from Arabidopsis Thaliana. Biochim. Biophys. Acta - Proteins Proteomics 2011, 1814, 1093-1105.

(9) Kondoh, M.; Terazima, M. Conformational and Intermolecular Interaction Dynamics of Photolyase/Cryptochrome Proteins Monitored by the Time-Resolved Diffusion Technique. Photochem. Photobiol. 2017, 93, 15-25.

(10) Bleul, R.; Ritzi-Lehnert, M.; Höth, J.; Scharpfenecker, N.; Frese, I.; Düchs, D.; Brunklaus, S.; Hansen-Hagge, T. E.; MeyerAlmes, F.-J.; Drese, K.S. Compact Cost-Efficient MicrofluidicsBased Stopped-Flow Device. Anal. Bioanal. Chem. 2011, 399, 1117-1125.
(11) Sarkar, S. S.; Udgaonkar, J. B.; Krishnamoorthy, G. Unfolding of a Small Protein Proceeds via Dry and Wet Globules and a Solvated Transition State. Biophys. J. 2013, 105, 2392-2402.

(12) Tang, G. Q.; Patel, S. S. Rapid Binding of T7 RNA Polymerase Is Followed by Simultaneous Bending and Opening of the Promoter DNA. Biochemistry 2006, 45, 4947-4956.

(13) Christie, J. M. Phototropin Blue-Light Receptors. Annu. Rev. Plant Biol. 2007, 58, 21-45.

(14) Swartz, T. E.; Corchnoy, S. B.; Christie, J. M.; Lewis, J. W.; Szundi, I.; Briggs, W. R.; Bogomolni, R. A. The Photocycle of a Flavin-Binding Domain of the Blue Light Photoreceptor Phototropin. J. Biol. Chem. 2001, 276, 36493-36500.

(15) Nakasone, Y.; Eitoku, T.; Matsuoka, D.; Tokutomi, S.; Terazima, M. Dynamics of Conformational Changes of Arabidopsis Phototropin 1 LOV2 with the Linker Domain. J. Mol. Biol. 2007, 367, 432-442.

(16) Nakasako, M.; Iwata, T.; Matsuoka, D.; Tokutomi, S. LightInduced Structural Changes of LOV Domain-Containing Polypeptides from Arabidopsis Phototropin 1 and 2 Studied by Small-Angle X-Ray Scattering. Biochemistry 2004, 43, 14881-14890.

(17) Kasahara, M.; Swartz, T.E.; Olney, M.A.; Onodera, A.; Mochizuki, N.; Fukuzawa, H.; Asamizu, E.; Tabata, S.; Kanegae, H.; Takano, M., et al., Photochemical Properties of the Flavin Mononucleotide-Binding Domains of the Phototropins from Arabidopsis, Rice, and Chlamydomonas Reinhardtii. Plant Physiol. 2002, 129, 762-773.

(18) Karayannis, M. I. Comparative Kinetic Study for Rate Constant Determination of the Reaction of Ascorbic Acid with 2,6-Dichlorophenolindophenol. Talanta 1976, 23, 27-30.

(19) Tonomura, B.; Nakatani, H.; Ohnishi, M.; Yamaguchi-Ito, J.; Hiromi, K. Test Reactions for a Stopped-Flow Apparatus: Reduction of 2,6-Dichlorophenolindophenol and Potassium Ferricyanide by l-Ascorbic Acid. Anal. Biochem. 1978, 84, 370-383.

(20) Takeda, K.; Nakasone, Y.; Zikihara, K.; Tokutomi, S.; Terazima, M. Dynamics of the Amino-Terminal and Carboxyl-Terminal Helices of Arabidopsis Phototropin 1 LOV2 Studied by the Transient Grating. J. Phys. Chem. B 2013, 117, 15606-15613.

(21) Peter, E.; Dick, B.; Baeurle, S. A. Mechanism of Signal Transduction of the LOV2-J $\alpha$ Photosensor from Avena Sativa. Nat. Commun. 2010, 1, 122.

(22) Jones, M. A.; Feeney, K. A.; Kelly, S. M.; Christie, J. M. Mutational Analysis of Phototropin 1 Provides Insights into the Mechanism Underlying LOV2 Signal Transmission. J. Biol. Chem. 2007, 282, 6405-6414. 


\section{For Table of Contents Only}

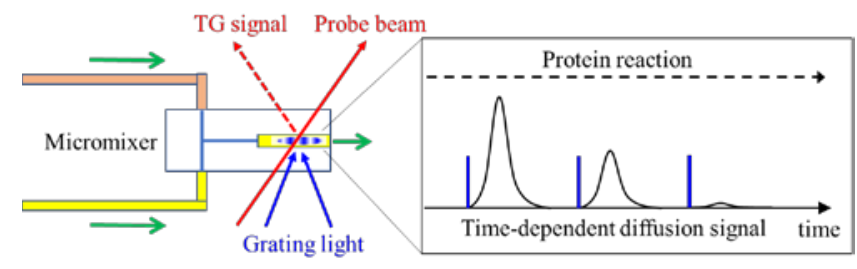

